\title{
SIFET-CBA Project
}

\author{
Mario A. Bochicchio, Antonella Longo, Federica Longo, Antonio Bernardo \\ Università del Salento, Italy, \{mario.bochicchio, antonella.longo, federica.longo, \\ antonio.bernardo\}@unile.it
}

\begin{abstract}
Innovating the Public Administration is not just the union of various tasks (innovating institutions, reengineering administrative processes and using innovative technologies) performed in isolation, because of the additional complexity coming from their coupling. Conceptual modelling techniques and rapid prototyping tools can help public managers and administrators to better understand the reciprocal dependencies between the various tasks and to bridge the gap between the different cultures and languages (juridical, organizational, managerial, information technology, ...) typical of the e-Government.
\end{abstract}

Keywords: conceptual modeling, system modeling, process innovation

\section{Introduction}

In recent years the diffusion of communication networks and distributed applications allowed the development of new interaction paradigms in Public Administration, under the collective name of e-Government, meant also as a way to organize public Administration for better serving citizens and enterprises on a comprehensive scale. As a consequence, the basic outline of an e-government vision has emerged and governments have taken promising steps to deploy egovernment services both in USA and in Europe [1]. Much remains to be done if this vision is to be broadly realized, to create innovative services within a coherent system of juridical and economical rules based on new technologies and concepts.

Innovating the Public Administration, in fact, is not just the union of various tasks (innovating institutions, reengineering administrative processes and using innovative technologies) performed in isolation, because of the additional complexity coming from their coupling [1]. The crucial point is to foster the consciousness of the holistic approach to integrate and extend models, design methodologies and techniques to face the new e-government challenges. This paper is focused on the innovation in Local Public Administrations and smallmedium public utilities (LPAs in the following), to give an integrated and transdisciplinary answer to the following questions: 
1. how to foster an effective e-gov. approach in the current context of "institutional uncertainty" about the role of the LPAs toward the citizen's community?

2. in the current panorama, what new options are imposed and what new tools and methodologies are given to LPAs to achieve a better performance and to improve their relationship with citizens?

In particular, referring to the latter question, the paper aims at two main goals:

a) how to involve the managers of LPAs in reshaping the organizational and operational structure of the same LPAs, adopting the ICT to improve their efficiency and effectiveness;

b) how to bridge the gap between the various "cultures" and languages (juridical, managerial, organizational, technological, ...) needed in the new e-gov. scenario.

We decided to orient our approach to Local Administrations because of their very poor performance in several key sectors: in Italy, for example, the official evasion figure of local taxation is around $30 \%$ while the fiscal contentious between citizens and LPA has reached a critical level [5]. Moreover, small and medium LPAs are often overwhelmed by outdated managers and bureaucratic employees culturally unable to manage the complexity of the new institutional and technological situation.

Among other LPAs, local public utilities are very problematic, like some Land Reclamation and Drainage Authorities of Apulia, that are in compulsory administration from 2004, because considered unable to properly manage the funding process (based on taxation) and to demonstrate the direct benefits produced to citizens. In the opinion of the authors, this "ineptitude" is directly related to the lack of (managerial, methodological, operational, juridical, ...) methodologies, tools and best practices to prefigure how normative and technological innovation can bridge the gap between the "paper based" government and the e-government

In the paper we propose an applied-research experience coming from the collaboration among the University of Salento and the Land Reclamation Authority of Arneo in the South-East of Apulia (Italy), with the aim at supporting the local utility to regain its role by increasing its performance and reducing the normative and informative asymmetries in taxation.

The paper is structured as follows. In Section 2, we present the context and the motivations at the root of our research. Section 3 discusses the reference framework we adopt and the main related tools. In Section 4 we present some results we achieved applying this framework to the Land Reclamation Authority of Arneo. Section 5 concludes the paper and depicts some further research developments. 


\section{Context}

In the perspective of the institutional dimension of technology innovation, the unclear distinction, widespread in the European legal tradition, between law and regulations creates uncertainty [6]. In fact, in the Law the decisional process takes place as authoritative form in the circle of command, with informal and uncontrollable behaviours. The result is a "mono-directional construction" aimed at reaching specific goals. The regulation, instead, is characterized by its practice, so it has conditional and "multidirectional" nature. It is based on the "what if" principle, with hypothetical not executive clarifications and it depends on the position of the single actor.

The use of ICT can produce radical improvements in administrative procedures, if it is supported by the optimization of the corresponding process. The normative techniques of regulation and simplification pursue the goal of "regulation in simplification" [8], which means:

- eliminating useless procedural steps unnecessary to decision making;

- reducing functional interferences between procedures regulated by different norms, unifying them in a unique procedural flow with one regulation;

- rationalizing the communication processes among the figures involved in decisions and creating a unique and consistent interface with the citizen;

- promoting the widespread access to decisions and their effects.

- The user centered approach in designing e-services and on-line applications is the corresponding facet in the software development community.

Actually, providing citizens with e-services is a hot research topic, and in particular researchers focus their attention on the implementation of a single point of access to public services and information, the development of integrated platforms, which will allow the public sector to provide citizens, businesses and other public authorities with information and public services structures. Examples of research and business applications and methods for integrating heterogeneous legacy information systems are extensively described in [3]. Nevertheless, the scenario is not homogeneous, and from an informal survey of the current context in the Southeast of Italy, we found:

- inconsistent and uncoordinated organizational growth with hundreds of processes overlapping in the years, with outdated organization solutions;

- incompatible ICT solutions and a correspondent inefficient usage of ICT

- lack of horizontal and vertical communication in the institutional structure.

- Other considerations related to the local taxes' management process contribute to better understand the poor quality of data owned by the Local Administration. In summary, our experience is that:

- Several sources may provide data to the system with different quality, according to the source (manual data input, data copied from system to system, etc.); 
- Data is often duplicated either due to the poor definition of the business logic (or bad bureaucratic processes) or to technical reasons, or simply for convenience.

- The semantic relationships among information don't exist, making it difficult to enforce the integrity constraints and to guarantee the data correctness.

\section{UWA+, methodology and tools}

The holistic approach in e-government creates new processes and scenarios, as they are highly knowledge intensive and they rely on the strict interaction between people and information technologies (IT in the following). These points imply that IT must reach the "heart" (the decisional level) of the administrative work, and not only its "hands" (the operational level). So the management of legal/administrative knowledge becomes a decisive driver in the administration. At the same time, designing governmental applications and services touches two issues: coupling concepts and systems and making use of standards. So, a right innovation methodology must combine Legal Drafting and engineering techniques, to develop normative and technological tools in an integrated fashion to reduce the gap of the normative asymmetry, cause of the informative asymmetry and of many inefficiencies.

Before the last Italian Constitutional reform (Law n. 3/2001) in LPAs the technological innovation (see [10]) has consisted of two alternative models: insourcing or outsourcing of ICT services [12]. The first approach has been subjected to unmodified informal behaviors and to authoritative bureaucratic formalism. In the latter case externalisation has made providers de-facto owners of data and knowledge, acquiring dominant position in IT innovation. In both cases LPAs have lacked any independent position in the adoption of new technologies and they have used (or have been used by) unidirectional approaches with no care about the delivery and the economic exploitation of information asset.

Nowadays LPAs, to exploit the autonomy, must "know themselves",: they must be conscious of their experiencies heritage, of their procedures and functionalities for delivering services, of their ability in understanding citizens needs, of their performance, just to be able to decide to change. Methodologies like UWA+ [13], extensively based on conceptual modeling techniques, can be very important to help LPAs to better define their internal structure, their processes and their information systems, and to manage and evolve it.

In this scenario, public utilities (LPAs in general) are very different from private ones because of the juridical nature of the relationship between citizens and the utilities: the services offered from the utilities are not an option for citizens, that are "forced" to pay for the received services. The form of the contribution is the same of a local tax, but in the new perspective of the constitutional reform. As a consequence, is not an option for the public utilities to 
correctly inform citizens about all aspects (both qualitative and quantitative) of the offered services.

This requires, the creation of an effective local taxation system, involving both legal and technological aspects, where fiscal questions are faced from different perspectives, in homogeneous legal and informative "environment", with the support of two complementary instruments called:

- TUnifET (Testo Unificato delle Entrate Tributarie, that is the Unified Sheet of Fiscal Incomes)

- SIFET (Sistema Informativo della Fiscalità e dei Tributi, that is the Fiscal Information System of the public utility).

- The description of the TUnifET, also reported in [14] for a similar case of study, is out of the scope of this paper.

- The creation of the SIFET for the CBA case of study is reported in the next subsection.

\subsection{SIFET}

In order to know itself and its territory and to achieve the tools and the abilities to measure its performance, the CBA must be conscious about the information and knowledge heritage owned inside, eventually completing and complementing it with external sources. The goal is to expose information owned inside, asking citizens and enterprises to complete and update it. New technologies (Internet, mobile, etc.) are very effective for this task, because they support the development of real-time/near-real-time services. To enable this instant access/instant response to take place, a blending of Ubiquitous Web applications with data from legacy archives and Information systems is needed, requiring design methodologies borrowed from both database and hypermedia communities.

With reference to taxation process operated by the CBA, the main goal has been the design and the implementation of the Office of Incomes, a center of real time incomes aggregation, to give CBA the chance to know its actual entrances and to plan the budget on "certified" data. The system for managing and facilitating the heterogeneous data transformation and integration is based on techniques of database integration design, while the design of the Web application for the citizen-side management of taxes is based on UWA+ framework [13] and on tools of fast prototyping [2]. The framework integrates a conceptual modeling methodology for designing web applications (UWA), with a tool for measuring KPIs (the HIGO grid) and with methods for integrating heterogeneous data sources. The user centred design methodology and the implementation of prototypes, generally recognized as very effective in improving the end user acceptance of the applications, aims to actively involve the CBA managers in the design of the SIFET. Data integration is constantly monitored by the Income Office, according to citizens' requests in the TunifET regulatory design. Beside, 
through the SIFET, citizens are initiated to use multichannel communication techniques with the Administration, in order to make the informative certainty easier, which is a necessary condition to reach the income certainty, vital for the Public Utility.

\section{Validating the approach: the SIFET-CBA project}

The Italian land reclamation and drainage authorities (consorzi di bonifica) are local public organizations, which main tasks are:

designing, implementing and maintaining public works concerning plumbing, drainage and land reclamation

improving the management of waters for different purposes (irrigation, dam , drainage management, ...)

Authority's operational expenses are split among the estates' owners of the area, according to the benefits achieved (or achievable) from the works made and maintained by the Authority. Yearly, the Authority requires each owner to pay for an amount determined by:

Estate's Land Register data of each owner;

Hydraulic technical land indexes (permeability, average slope, ...) of each land parcel;

Authority's maintained works' usage

The Consorzio di Bonifica dell'Arneo (CBA in the following, literarily: Land Reclamation and Drainage Authority of Arneo), is a public organization based in Nardò, in the South-East part of Italy. It employs 68 people, manages 250.000 hectares and 200.000 estates in Salento area, and yearly issues 180.000 notices for about $15 \mathrm{M€} /$ year, but in the last operation year (2004) 95\% of them were claimed with open questions of law.

So the aim of the project has been to support CBA in reducing the information asymmetry between the Authority and land owners through an extensive use of new technologies.

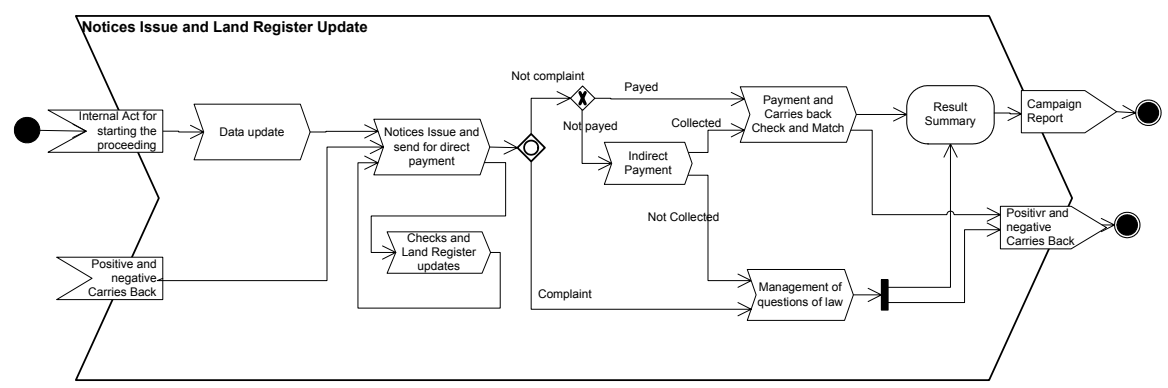

Fig. 1. Notices Issue and Land Register Update Process overview 
In order to allow management to evaluate the impact of their choices in the egov scenario, we shared the UWA+ framework main outcomes and implemented a working prototype. Thus a further result has been the formalization of the AS-IS and TO-BE process of notice issue and collection and the definition of KPIs according to the different stakeholders in order to evaluate the performances in relation to the different choices.

Today in CBA the process starts with a request from the Authority board to the EDP Manager to issue payment notices (Figure 1). The first step is the calculation of the due amount for each estate insisting on the pertinent area; since necessary info is on paper, EDP workers manually update info into an AS400 system. This data are

contained in a document called Parcel Sheet, about the cadastral income, the land income, technical indicators about the works intensity, the hydraulic behaviour index, the land stock index

about hydraulic works related to the estate, which, if well maintained by the Authority, improve the estate value

about owner's personal data, payments, refunds and sanctions.

Then payment notices are printed and directly sent to each owner, to be paid within a deadline. The not-paid notices are collected by to a debt collector for indirect payment.

Payments and carries back are matched with collected payments notices and positive or negative differences are notified to owners.

An office counter is always available to receive complaints and update the estate' status in the Land Register during the direct and indirect issue and collection period.

For lack of space, we only show diagrams of the sub process about notice issue for the direct payment. Figure 3 displays the AS -IS assembly line diagram. It is made up with the activity diagram in the upper part, showing actors performing the different activities, the automated activities (with the computer icon), and the involved information resources in the lower part. The two are connected through $\mathrm{read} / \mathrm{write}$ data links with the related activities (the black points show read data, white points are information written during the activity).

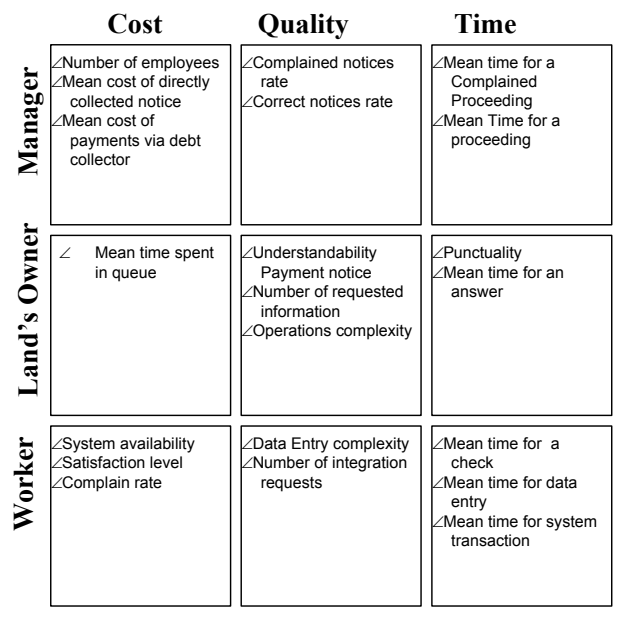

Fig. 2. Stakeholders KPIs according to HIGO approach 


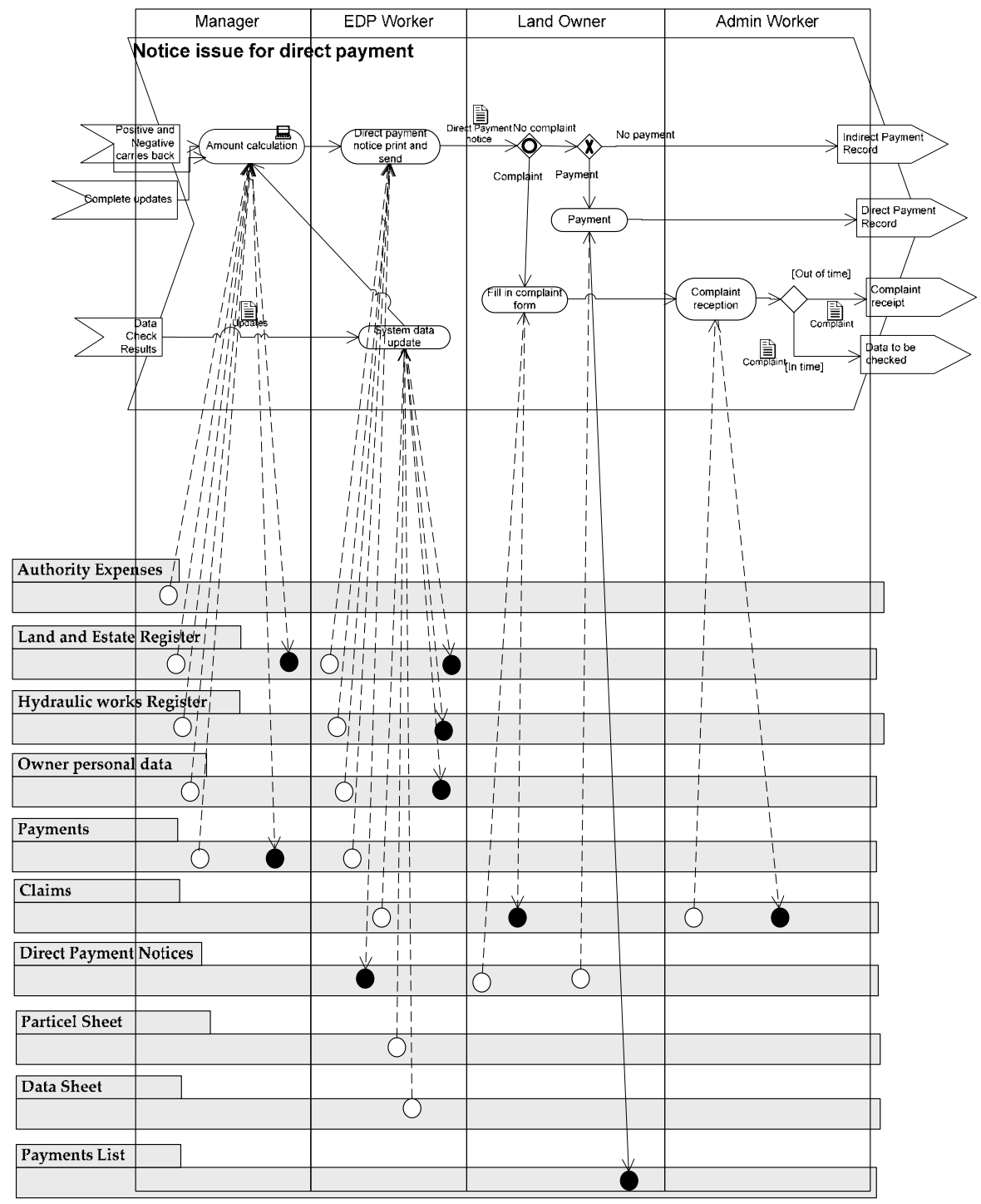

Fig. 3. Assembly line diagram of the AS IS notice issue for direct payment process

According to Higo grid, KPIs have been defined in the categories of Cost, Quality and Time for each stakeholder, worker, land's owner and manager, as shown in Figure 2. 
Table 1. Target objectives for Manager's KPIs

\begin{tabular}{|c|c|c|}
\hline \multicolumn{2}{|c|}{ Manager Indicators } & \multirow{2}{*}{ To-Be Objective } \\
\hline Category & Indicator & \\
\hline \multirow{3}{*}{ Cost } & Number of Employees (FTE) & Reduce the work load per employee by $30 \%$ \\
\hline & $\begin{array}{l}\text { Mean cost of directly collected } \\
\text { notice }\end{array}$ & Reduce the front end work hours by $50 \%$. \\
\hline & $\begin{array}{l}\text { Mean cost of a notice via debt } \\
\text { collector }\end{array}$ & Reduce the front end work hours by $50 \%$ \\
\hline \multirow[b]{2}{*}{ Time } & Mean Time for a proceeding & The whole mean time must be reduced by $50 \%$ \\
\hline & $\begin{array}{l}\text { Mean time for a Complained } \\
\text { Proceeding }\end{array}$ & Reduce the time for a practice by $30 \%$, \\
\hline \multirow{2}{*}{ Quality } & Correct notices rate & Improve notices correctness by $80 \%$ \\
\hline & Complained notices rate & Reduce the complained notice rate by $50 \%$ \\
\hline
\end{tabular}

In order to improve the process efficiency and the relationship quality with customers, the new target values have been defined, and a new process has been designed, supported by Web tools. As an example Table 1 shows KPIs target values for the Manager's stakeholder,

The new process aims at balancing the information asymmetry towards the user, let him be the manager of his information, update his data and provide land's owner with motivations of his notice amount.

Figure 4 shows the Assembly line diagram of the TO-BE notice issue for direct payment process. It has been designed to meet the following goals:

- to provide customers with an always and everywhere available counter in order to reduce litigations related to not updated data

- to provide customers with a tool enabling them to manage their information and payments

- to update the Land Register, which must be accessible in real time for calculation and checks 


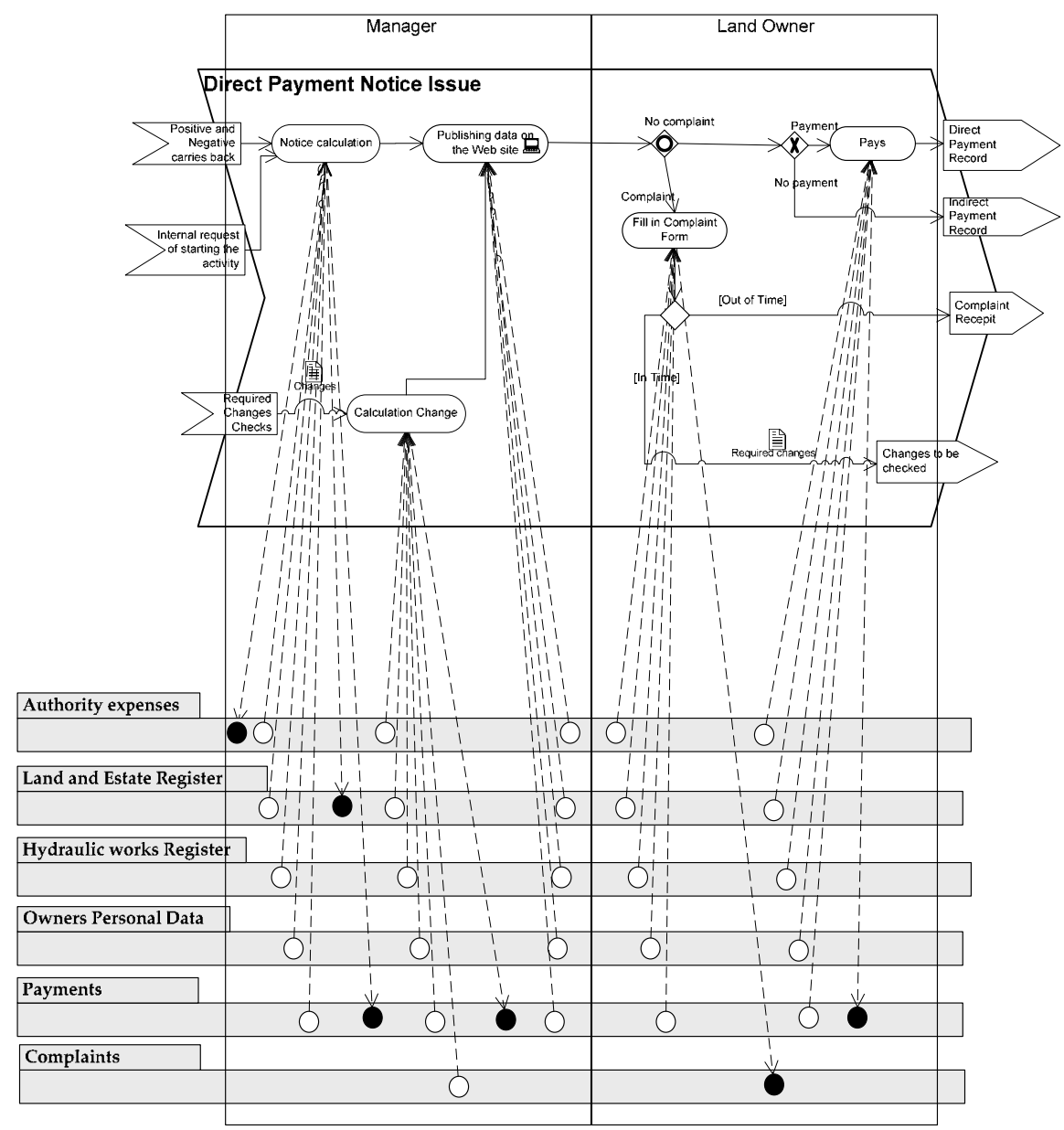

Fig. 4. Assembly line diagram of the TO-BE notice issue for direct payment process

The conceptual model of the Web application has been designed using UWA approach. In Figure 5 the hyperbase in the large is displayed, showing the main information entity types and their semantic relationships. Entity types are straight related with the information resources in the assembly lines. Figure 6 shows the hyperbase in the small of the Building entity type where attributes are straight related with the data model, necessary to implement the prototype using a fast prototyping tool. 


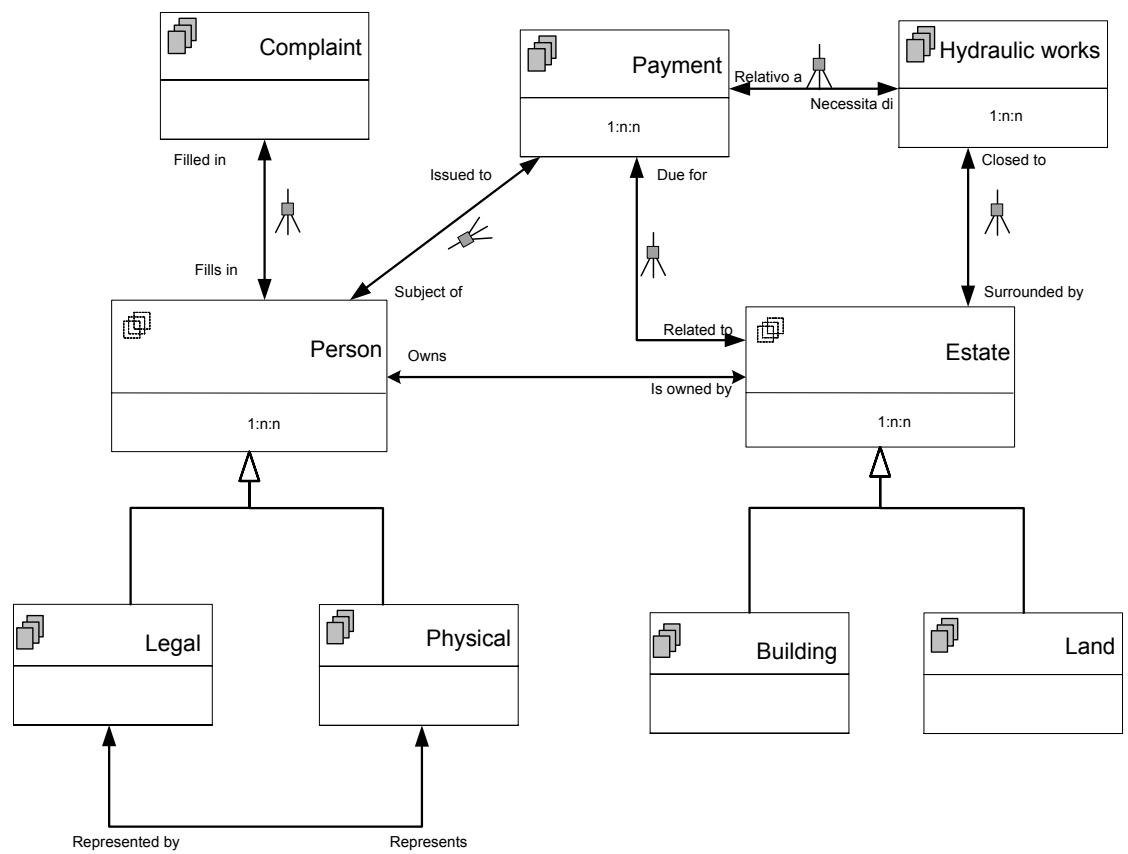

Fig. 5. Hyperbase in the large of the Web Application supporting the Notice issue and payment process

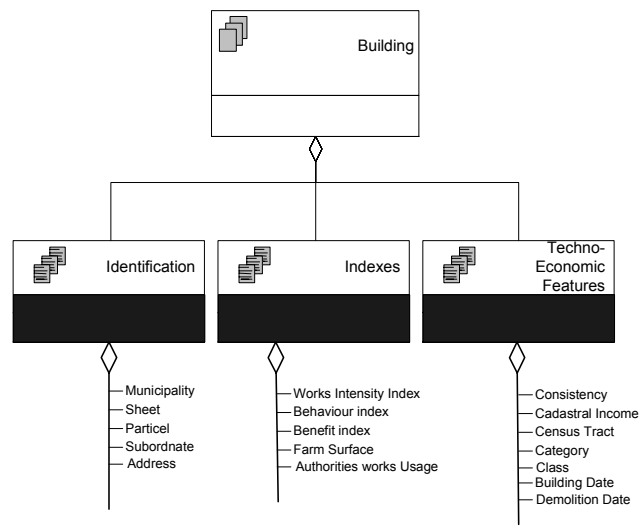

Fig. 6. Hyperbase in the small of the Building Entity Type

The result is shown in Figure 7: the screenshot displays the land owner personal web page, with his personal information, his buildings, his lands, the notices to be paid online and the ones already sent to the debt collector. 
The prototype has been discussed and tested with CBA employees, simulating different user scenarios and evaluating with the management the impact of the diverse choices.

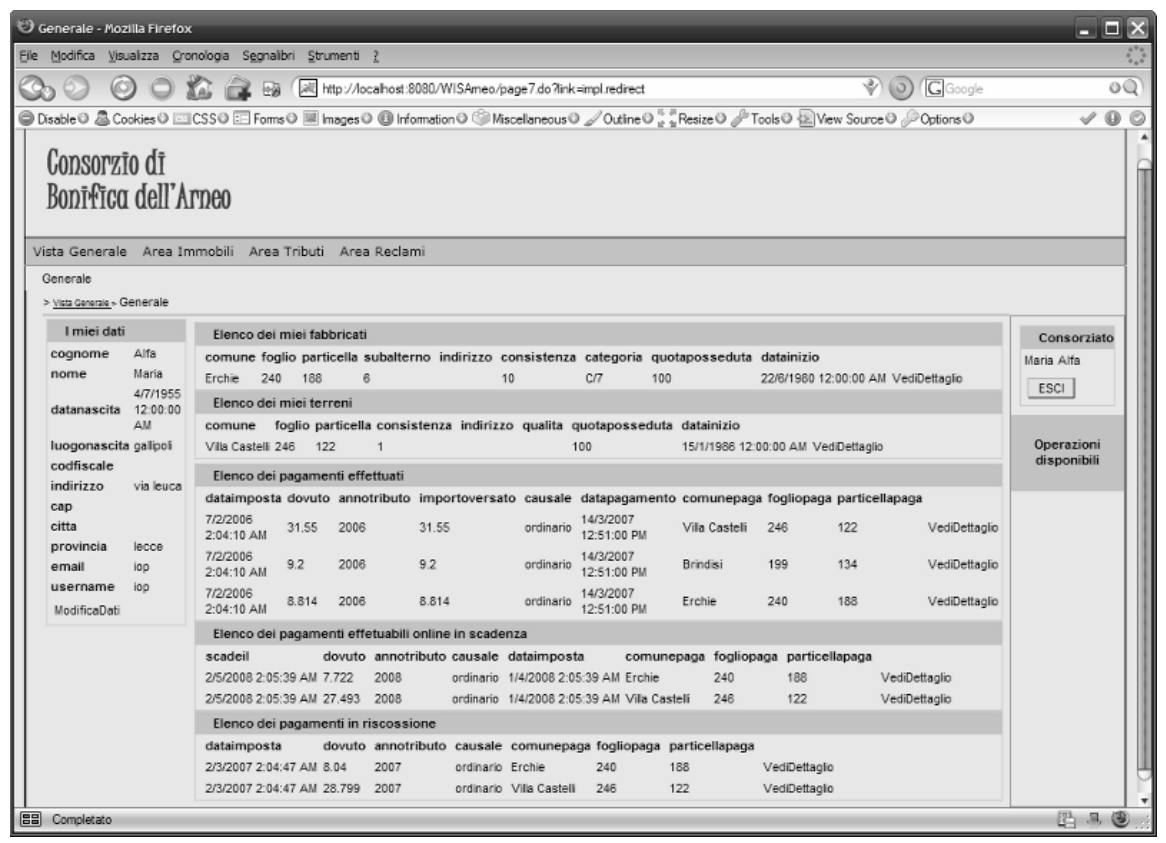

Fig. 7. A screenshot of the Web Application supporting the notice issue process.

\section{Conclusions and further works}

The interdisciplinary experience of CBA project has promoted the institutional, normative and organizational learning process. In this e-government experience CBA has not been "buyer of innovation", but "author and actor of institutional innovation" in the normative and informative scenario of technology usage. So, instead of bearing the increase of knowledge, learning, discipline and adaptation costs, the CBA finds out the relativity of the e-government impact in an ongoing transforming context, like the Constitutional Italian one, and, in the specific sample of local taxation, it combines innovation and the reconstruction of trust in the relationship with citizens, on the ground of information circulation and availability. The results concern

The definition of clear lexicon and semantic in the regulations with taxpayer's relationship

Positive financial benefits without additional costs

The elimination of unwanted effects, like fiscal trial 
- The main advantages perceived by CBA's board have been:

- learning by doing for employees involved in the project, and knowledge transfer from the project team to the rest of employees;

- better aptitude to innovation;

- savings and reorganization of local taxation.

We are planning to extend the empirical validation of the model and the tools in order to tune and customize them to different contexts and sizes. Moreover this experience of holistic model can be extended from the local taxation to other functions in the LPA, having already verified the citizens' goodwill to pay according to indicators of regulation and reorganization of informative processes.

\section{References}

1. Lenk k., Traunmüller R., Electronic Government: Where Are We Heading?, in Traunmüller R., Lenk K. (Eds), EGOV 2002, LNCS 2456, pp. 1-9, 2002, Springer-Verlag, Heidelberg 2002.

2. UWA (Ubiquitous Web Applications) Project: http://www.uwaproject.org.

3. Bochicchio M. A., Longo A.: "An Effective Approach to Reduce the "Avalanche Effect" in the Management of Fiscal Data in Local Public Administration. ICSM 2002: 560- 567

4. Bochicchio M., Longo A.: "Data Cleansing for Fiscal Services: the Taviano Project", in ICEIS 2003, Angers, April 2003

5. Ministero dell'Interno, Analisi Gestionale e Finanziaria degli Enti Locali (Ufficio Studi della Direzione Centrale della Finanza Locale), Settembre 2002.

6. Graham G.A., Regulatory Administration, in Graham G.A., Reining jr. H.F. (eds.), Regulatory administration, Wiley and Sons, New York, 1943, 16

7. Dipartimento della Funzione Pubblica, Proposte per il cambiamento nelle pubbliche amministrazioni, Rubettino, Soveria Mannelli, 2002

8. OECD, Regulatory quality and public sector reform, Puma/reg (97), 1, Paris, 1997

9. Salmon P., Decentralization as an Incentive Scheme, in Oxford Review of Economic Policy, vol. 3, n. 2, 1987, pp. 24-43

10. Morgan G. Images, Trad. italiana, Franco Angeli, Milano. 1995

11. Giarda P., Le regole del federalismo fiscale nell'articolo 119: un economista di fronte alal nuova Costituzione, JEL Classification. H7, Working Papers No. 115/2001, Jan. 2002, 11

12. Natalini A., Le semplificazioni amministrative, Il Mulino, Bologna, 2002

13. Bochicchio M. A., Longo A., UWA+: bridging Web systems design and Business process modeling, workshop on "Hypermedia Development \& Web Engineering Principles and Techniques: Put them in use.", HT04, http://www.ht04.org/workshops/WebEngineering/HT04WE Bochicchio.pdf

14. A. Longo, M. Bochicchio, M. Carducci, "Reducing Normative and Informative Asymmetries In Fiscal Management for Local Administration", in "Digital Communities in a Networked Society e-Commerce, e-Business and e-Government", Ed. R. Suomi, C. Passos, M. Mendes, Kluwier Academic Publishers ISBN 1-4020-7795-5, pp. 13-24. 